\title{
Sub-Cellular Network Analysis of Ryanodine Receptor Positioning in Control and Phosphorylated States
}

\author{
Ismail M. Khater ${ }^{1}$, David R.L. Scriven ${ }^{2}$, Edwin D.W. Moore ${ }^{2}$, Ghassan Hamarneh ${ }^{1}$ \\ ${ }^{1}$ Medical Image Analysis Lab, Simon Fraser University, Canada \\ ${ }^{2}$ Department of Cellular and Physiological Sciences, University of British Columbia, Canada
}

\begin{abstract}
Type 2 ryanodine receptors $(R y R 2)$ are large ( mass $=2.2$ MegaDalton) proteins expressed in heart cells that mediate the release of calcium ions $\left(\mathrm{Ca}^{2+}\right)$ that, in turn, modulate contraction of the heart. In this work, we analyze the sub-cellular spatial distribution of RyR2 using data from superresolution microscopy, an imaging technique that allows highly accurate positioning $(<10 \mathrm{~nm}$ in $x$ and $y ;<40$ $n m$ in z) of the RyR2 within the heart muscle cell. In particular, we present the first work to examine network measures of extracted RyR2 locations to examine the clustering behaviour of these channels. We collected images from two groups of healthy cardiac cells; the control consisted of 8 cells, while the second group of 8 cells were treated with a chemical cocktail to phosphorylate the RyR2 and to inhibit dephosphorylation. We examined the classification accuracy (using random forest classifier) and the group differences (using Mann-Whitney statistical test and Bonferroni multiple comparison correction) based on several network measures, at multiple proximity thresholds. Several network measures we examined revealed features (e.g. network clustering) that enabled us to differentiate between these two populations $(p<0.00045)$ with high classification accuracy ( $>95 \%$ at proximity thresholds 200 and $250 \mathrm{~nm}$ ). Our findings may help in better understanding $\mathrm{Ca}^{2+}$ signaling during contraction and give insight into the changes that underlie its regulation.
\end{abstract}

\section{Introduction}

The process of calcium release is essential for subcellular dynamic functions such as excitation-contraction (EC) coupling in cardiac myocytes. The ryanodine receptors (RyRs) are a class of large protein channels responsible for mediating the rapid release of calcium ions $\left(\mathrm{Ca}^{2+}\right)$ from intracellular stores into the cytosol [1]. There are multiple isoforms of RyR proteins: RyR1 is primarily expressed in skeletal tissues. RyR2 is primarily expressed in cardiac myocytes and RyR3 is widely expressed in the brain. In this paper, we focus on cardiac cells and on RyR2 proteins in the dyad. The dyad is the structural element formed by the close apposition of the membranes of the sarcolemma (either surface or t-tubule) and the junctional sarcoplasmic reticulum (jSR) [2]. RyR2 proteins are distributed on the jSR membrane opposite the L-type channels in the sarcolemma.

The principal determinant of the force of cardiac contraction is the amount of $\mathrm{Ca}^{2+}$ released from the sarcoplasmic reticulum, and this is controlled in part by how RyR2 are distributed relative to each other. Electron tomography has demonstrated that the RyR2 distribution can be altered by physiological factors, such as phosphorylation (the fight or flight response) [3].

The recent development of superresolution microscopy (also called nanoscopy), such as direct stochastic optical reconstruction microscopy $d$ STORM, can bring new insight into cardiac cell biology. At a high level, $d$ STORM nanoscopy offers single molecule localization and allows for nanometer-precision identification of RyR2 locations, which in turn enables us to study their arrangements [4]. This will lead to a better understanding of $\mathrm{Ca}^{2+}$ signaling during cardiac E-C coupling.

Superresolution microscopy has been performed on cardiac myocytes [4] and [5] but neither in 3D nor using network measures. In this work, we quantitatively study the 3D spatial arrangements of a large number of RyR2 blinks from $d$ STORM data while making no a priori assumptions about the underlying distribution. We do so by representing the RyR2 as a complex protein network, analysing its topological features, and determining whether those features are altered by factors such as phosphorylation. Complex networks have been analysed at the macro-scale using diffusion MRI for brain images [6,7], but similar analyses at the sub-cellular level for cardiac proteins are yet to be performed. Other quantitative analysis of superresolution microscopy data were performed by Owen et al. [8] and [9]. However, they studied constellations of T-cells (not cardiac-related proteins) using second order statistics (Ripley's function) and not complex network features or were 
Table 1. Survey of most related work. Dim: dimensionality; Analysis: quantitative/ qualitative; ML: machine learning; Net: network measures; and Res: resolution/scale.

\begin{tabular}{|c|c|c|c|c|c|c|c|}
\hline Reference & Dim & Modality & ML & Net & Field & Analysis & Res \\
\hline \hline$[10]$ & 2D & elec./confocal microscopy & No & No & cardiac (RyR2) & Qualit./Visual & $\mu \mathrm{m}$ \\
{$[8]$} & 2D & superresolution & No & No & T-Cells & Quantitative & $\mathrm{nm}$ \\
{$[9]$} & 3D & superresolution & No & No & T-Cells & Quantitative & $\mathrm{nm}$ \\
{$[1]$} & 3D & electron cryomicroscopy & No & No & cardiac (RyR1) & Qualitative & $\AA$ \\
{$[4]$} & 3D & superresolution & No & No & cardiac (RyR2,JPH2) & Survey & $\mathrm{nm}$ \\
{$[6,7]$} & 3D & functional/structural MRI & No & Yes & brain & Quantitative & $\mathrm{mm}$ \\
ours & 3D & superresolution & Yes & Yes & cardiac (RyR2) & Quantitative & $\mathrm{nm}$ \\
\hline
\end{tabular}

limited to 2D.

To the best of our knowledge, our work is the first to study 3D configurations and interactions of RyR2 proteins in cardiac cells via quantitative analysis of complex networks (Table 1). In particular, we have analysed two populations of RyR2, control and phosphorylated, as complex networks and extracted network measures as features for machine learning (classification) techniques.

\section{Method}

Overview: Our hypothesis is that there are differences in the arrangement of RyR2 between the two populations (control vs. phosphorylated). To examine this hypothesis, at a high level (Figure 1), we acquired $d$ STORM images from both populations, extracted 3D locations (point clouds) of RyR2, encoded the point cloud as a complex biological network, and extracted network measures. We examined the discrimination ability of these features by training a machine learning classifier and performing statistical analysis of group differences.

Data Collection: We collected images from two groups of healthy cardiac cells. The control group consisted of 8 cells while the second group of 8 cells were treated with a chemical cocktail to phosphorylate the RyR2 and to inhibit dephosphorylation [3]. Phosphorylation increases cardiac contractility by, in part, increasing the likelihood that the RyR2 channel will open, but how this is accomplished is poorly understood. We used $d$ STORM to image RyR 2 proteins [11], from which we constructed a 3D point-cloud. To limit the computational times we first segmented the images to isolate Z-lines ( 9 from the control cells and 14 from those that were phosphorylated; between 30,000 and 65,000 blinks each), which is where jSR and RyR2 are primarily located.

Network Construction: Given the locations of all the RyR2 proteins, we constructed a weighted undirected graph $G=(V, E)$, with a set of vertices $V$ that represent the RyR2 proteins, and a set of edges $E$ that represented the interaction between the RyR2s, where each edge is a pair of vertices $\{u, v\}$. An edge is connected between a pair of proteins $(i, j) \in V$ only if the Euclidean distance between the nodes is less than a graph connectivity prox-

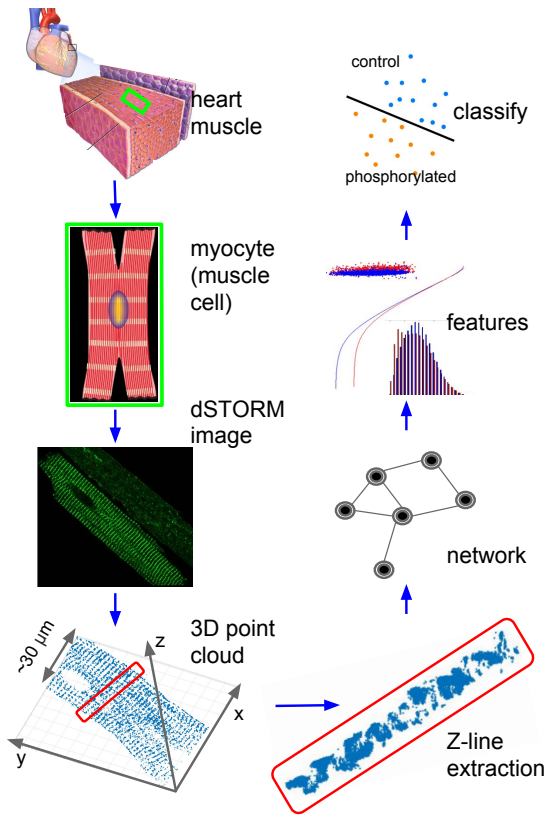

Figure 1. Proposed RyR2 biological network analysis and classification pipeline.

imity threshold $T$.

Network Feature Extraction: To compare the connectivity patterns of the RyR2 in both populations (control and phosphorylated), we extracted 16 network measures, summarized in (Table 2), at different thresholds: $T \in\{$ $50,75,100,150,200,250,300\} \mathrm{nm}$, i.e. we represent each network by a $7 \times 16=112$-dimensional feature vector. These measures capture different aspects of clustering and community structures [12]. Since there are multiple antibody binding sites on an individual RyR2, which is $\sim 30 \times 30 \times 12 \mathrm{~nm}$, we set a lower limit of $50 \mathrm{~nm}$ to avoid identifying individual RyR2 as multiple nodes. In order to handle the network analysis we used the Pajek-XLL Software Package ${ }^{1}$.

Classification and Statistical Analysis: We used the extracted features for classification, using a random forest (RF) classifier, with 10-fold cross-validation. We also performed the Mann-Whitney non-parametric statistical test

\footnotetext{
${ }^{1}$ http://mrvar.fdv.uni-lj.si/pajek/
} 
Table 2. Complex network measures used in this work.

\begin{tabular}{|c|c|}
\hline Network measure & Description \\
\hline $\begin{array}{r}\text { numNodes } \\
\text { numEdges } \\
\text { avgDegree } \\
\text { netTransitivity } \\
\text { netModularity } \\
\text { WSClustCoef }\end{array}$ & $\begin{array}{l}\text { Number of nodes in the network } \\
\text { Number of edges in the network } \\
\text { Average network degree } \\
\text { Network Transitivity } \\
\text { Network Modularity } \\
\text { Watts-Strogatz clustering coefficient }\end{array}$ \\
\hline $\begin{array}{r}\text { densityLoops } \\
\text { densityNoLoops }\end{array}$ & $\begin{array}{l}\text { Network density (loops allowed) } \\
\text { Network density (no loops allowed) }\end{array}$ \\
\hline $\begin{array}{r}\text { degCentralization } \\
\text { degCentLowest } \\
\text { degCentHighest } \\
\text { degCentAverage } \\
\text { degCentStdDev }\end{array}$ & $\begin{array}{l}\text { All-node average degree centralization } \\
\text { Lowest degree centralization } \\
\text { Highest degree centralization } \\
\text { Average degree centralization } \\
\text { Standard deviation of degree } \\
\text { centralization values }\end{array}$ \\
\hline $\begin{array}{r}\text { numConnComp } \\
\text { sizeLargestConnComp } \\
\text { percentLargeConnComp }\end{array}$ & $\begin{array}{l}\text { Number of weak connected components } \\
\text { (i.e. number of clusters) } \\
\text { Size of the largest connected component } \\
\text { Percentage of number of nodes in the } \\
\text { largest connected component to total } \\
\text { number of nodes in network }\end{array}$ \\
\hline
\end{tabular}

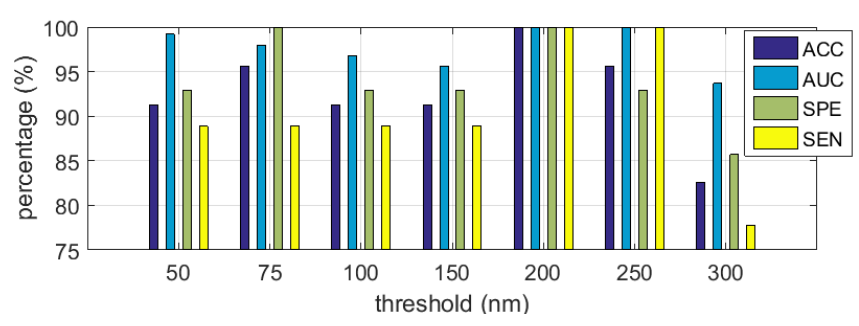

Figure 2. RyR2 network classification accuracy (ACC\%), area under the ROC curve (AUC\%), specificity (SPE\%) and sensitivity (SEN\%).

to examine group differences.

\section{Results and Discussion}

In our first experiment, we trained the RF classifier (with 100 trees) to distinguish between control vs. phosphorylated cells, using features extracted at different network thresholds $T$. Figure 2 depicts the classification accuracy (ACC), area under the ROC curve (AUC), specificity (SPE), and sensitivity (SEN) values.

It is clear from Figure 2 that we achieve excellent classification performance near $200 \mathrm{~nm}$ (accuracy 100\% at $T=200 \mathrm{~nm}$ and $95.7 \%$ at $T=250 \mathrm{~nm}$ ). At higher thresholds $T>300 \mathrm{~nm}$, we see a relatively lower classification performance $(\sim 82.6 \%)$, which is expected because excessive merging of RyR2 clusters occur as we increase the proximity threshold, thus diminishing any group-specific clustering characteristics. At thresholds $T \leq 200 \mathrm{~nm}$, the classification results also deteriorate but remain relatively acceptable (accuracy 91-95.7\%), which may corroborate other findings linking heart pathologies (e.g. heart failure under extreme stress) with reduction in the size of the dyad at comparable scales [2].

In our second experiment, we sought to discover which

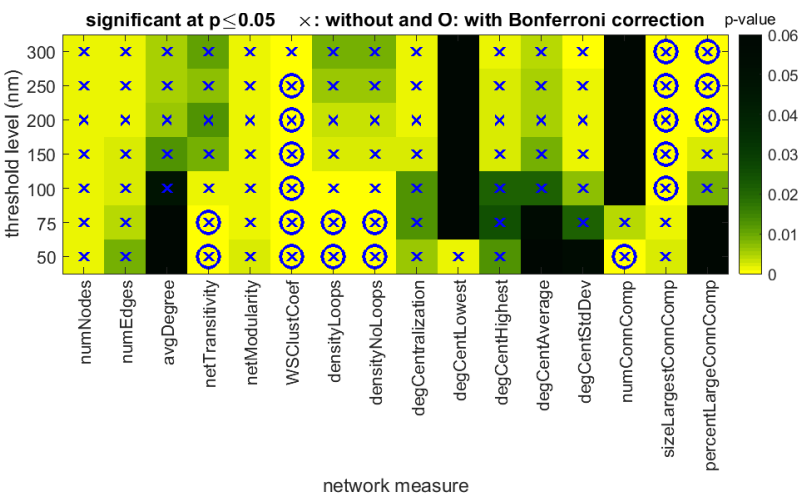

Figure 3. Discriminability of network measures at multiple thresholds. Colors correspond to $p$ values. Brighter yellow indicates stronger differences between groups. Measures that showed statistically significant differences at $p \leq 0.05$ are indicated by $\times$ and by $\bigcirc$ with Bonferroni correction (i.e. $p \leq 0.05 /(16 \times 7)=\sim 0.00045$ for 16 features and 7 thresholds).

features were discriminatory at various thresholds $T$. Since the feature sets of the two groups (control vs. phosphorylated) are not normally distributed, as per the Kolmogorov-Smirnov normality test $(p \leq 0.0001)$, we used the non-parametric Mann-Whitney statistical test to evaluate the null hypothesis that the network features of the two cell types followed the same distribution. Figure 3 shows which features and at which thresholds there were statistically significant differences between the two classes, evaluated at significance level $p \leq 0.05$. Since we performed multiple pairwise tests $(7$ thresholds $\times 16$ network measures) on a single dataset, we show the results with and without the application of Bonferroni multiple comparison correction; the latter used to reduce the chance of obtaining false-positive results (type I errors).

From Figure 3 we see that the most discriminatory features $(p<0.00045)$ at low thresholds $(50,75$ $\mathrm{nm})$ are related to network density (densityLoops, densityNoLoops) and number of connected components (numConnComp). The size of the largest connected component (sizeLargestConnComp), on the other hand, becomes discriminatory at thresholds $(T>100$ $\mathrm{nm}$ ). The clustering coefficient (WSClustCoef) proved discriminatory at a wide range of thresholds $(50 \leq T \leq$ $250 \mathrm{~nm}$ ). For example, taking a closer look at the values of numConnComp and sizeLargestConnComp at varying thresholds (Figure 4), we note that as we increase the threshold, RyR2 clusters merge and the number of clusters (numConnComp) decrease (Figure 4-a), making it harder to discriminate between control vs. phosphorylated at higher thresholds. On the other hand, the feature capturing the size of the largest cluster (sizeLargestConnComp) becomes more discriminatory at larger thresholds (Figure 4-b).

Given the importance of RyR2 clustering-related net- 
(a)
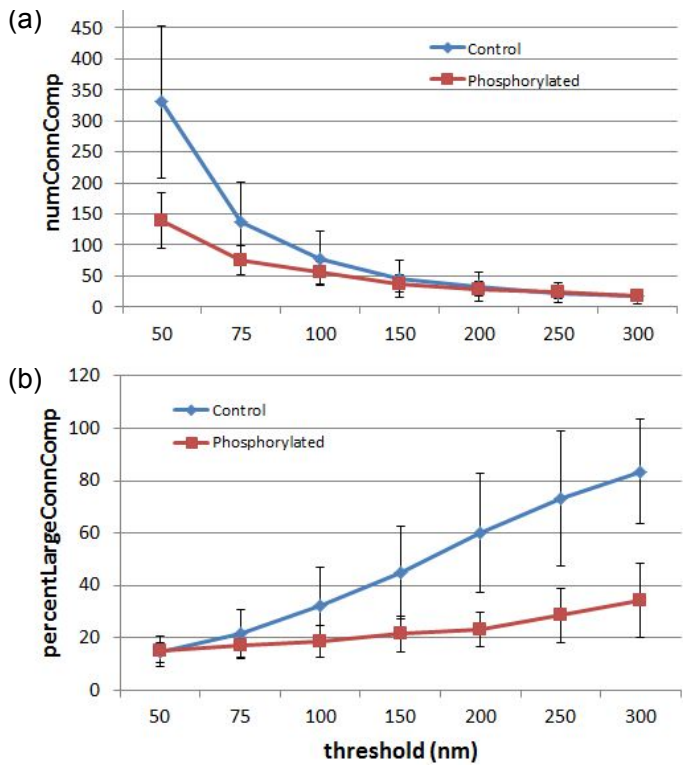

Figure 4. A closer look at the behaviour of RyR2 clustering. As the number of connected components (numConnComp) become less discriminatory at high thresholds (a), the size of the largest connected component (percentLargeConnComp) becomes discriminatory (b).

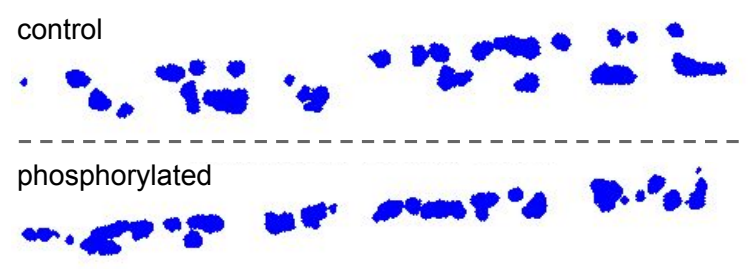

Figure 5. Visualization of RyR2 clusters at $\mathrm{T}=100 \mathrm{~nm}$ in Z-lines of a control cardiac cell (top) vs. phosphorylated cardiac cells (bottom).

work measures in describing group differences, we visually observed how the behaviour of RyR2 clustering changes between control and phosphorylated states, Figure 5. Note how in the phosphorylated state (Figure 5-bottom) the RyR2 clusters are beginning to merge, forming a more compact chain-like structure along the Z-line, whereas the RyR2 clusters in control group are more disperse (Figure 5-top).

Electron tomography has identified the position of individual RyR2 proteins and changes in their distribution in response to phosphorylation (Asghari et al. [3]). While each tomogram is limited to a single dyad or to a portion of it (400 nm or less), our network analyses can assess clustering at large spatial scales, thousands of $\mathrm{nm}$ or more. This has allowed us to determine that phosphorylation promotes the formation of large RyR2 clusters from smaller ones. This has both complemented and extended the observations of Asghari et al. [3].

\section{Conclusions}

We have provided the first quantitative analysis of RyR2 proteins in cardiac cells using network analysis and demonstrate that this may be a useful technique for analysing superresolution immunofluorescence data without making any assumptions about the underlying molecular distribution. By performing statistical analyses and machine learning based classification of complex network features, we observed clustering differences between two groups of cardiac cells, control vs. phosphorylated. Future work, will involve studying the morphology of the individual RyR2 clusters on a larger number of datasets.

\section{References}

[1] Zalk R, Clarke O, des Georges A, Grassucci R, Reiken S, Mancia F, Hendrickson W, Frank J, Marks A. Structure of a mammalian ryanodine receptor. Nature 2015;517:44-49.

[2] Scriven D, Asghari P, Moore E. Microarchitecture of the dyad. Cardiovascular Research 2013;98:169-176.

[3] Asghari P, Scriven D, Sanatani S, Gandhi S, Campbell A, Moore E. Nonuniform and variable arrangements of ryanodine receptors within mammalian ventricular couplons. Circulation research 2014;115:252-262.

[4] Kohl T, Westphal V, Hell S, Lehnart S. Superresolution microscopy in heart - cardiac nanoscopy. Journal of Molecular and Cellular Cardiology 2013;58:13-21.

[5] Baddeley D, Jayasinghe I, Lam L, Rossberger S, Cannell M, Soeller C. Optical single-channel resolution imaging of the ryanodine receptor distribution in rat cardiac myocytes. Proceedings of the National Academy of Sciences 2009; 106:22275-22280.

[6] Bullmore E, Sporns O. Complex brain networks: graph theoretical analysis of structural and functional systems. Nature Reviews Neuroscience 2009;10:186-198.

[7] Rubinov M, Sporns O. Complex network measures of brain connectivity: Uses and interpretations. NeuroImage 2010; 52:1059-1069.

[8] Owen D, C. R, Rossy J, Magenau A, Williamson D, Rodriguez M, Gaus K. PALM imaging and cluster analysis of protein heterogeneity at the cell surface. Journal of biophotonics 2010;3:446-454.

[9] Owen D, Williamson D, Boelen L, Magenau A, Rossy J, Gaus K. Quantitative analysis of three-dimensional fluorescence localization microscopy data. Biophysical Journal 2013;105:L05-L07.

[10] Lukyanenko V, Ziman A, Lukyanenko A, Salnikov V, Lederer W. Functional groups of ryanodine receptors in rat ventricular cells. 2007;583:251-269.

[11] Tafteh R, Scriven D, Moore E, Chou K. Single molecule localization deep within thick cells; a novel super-resolution microscope. Journal of Biophotonics 2016;9:155-160.

[12] Newman M. The structure and function of complex networks. SIAM review 2003;45:167-256. 